Cinémas

Revue d'études cinématographiques

Journal of Film Studies

\title{
Vers une théorie du récit automatique
}

\section{Georges Nault}

Volume 2, numéro 1, automne 1991

Le Scénario

URI : https://id.erudit.org/iderudit/1001055ar

DOI : https://doi.org/10.7202/1001055ar

Aller au sommaire du numéro

\section{Éditeur(s)}

Cinémas

ISSN

1181-6945 (imprimé)

1705-6500 (numérique)

Découvrir la revue

Citer cet article

Nault, G. (1991). Vers une théorie du récit automatique. Cinémas, 2(1), 137-148. https://doi.org/10.7202/1001055ar

\section{Résumé de l'article}

Au-delà des méthodes traditionnelles d'écriture de scénarios, on peut vraisemblablement concevoir une utilisation des ordinateurs modernes et des techniques particulières à l'intelligence artificielle à des fins de support à la créativité et à l'imagination. L'auteur décrit comment il a pu construire une formalisation des théories narratives du récit suffisamment stricte et rigoureuse pour permettre l'élaboration et l'implantation d'un système de génération automatique de récits. Ce système expérimental repose en grande partie sur la capacité particulière aux ordinateurs de pouvoir générer et évaluer une grand nombre d'alternatives de récits possibles et cela dans des temps relativement courts. Un tel système se veut une aide à la création pour un éventuel scénariste et ne prétend aucunement remplacer l'humain dans sa tâche première de création. 


\title{
Vers une théorie du récit automatique
}

\section{Georges Nault}

\begin{abstract}
RÉSUMÉ
Au-delà des méthodes traditionnelles d'écriture de scénarios, on peut vraisemblablement concevoir une utilisation des ordinateurs modernes et des techniques particulières à l'intelligence artificielle à des fins de support à la créativité et à l'imagination. L'auteur décrit comment il a pu construire une formalisation des théories narratives du récit suffisamment stricte et rigoureuse pour permettre l'élaboration et l'implantation d'un système de génération automatique de récits. $\mathrm{Ce}$ système expérimental repose en grande partie sur la capacité particulière aux ordinateurs de pouvoir générer et évaluer une grand nombre d'alternatives de récits possibles et cela dans des temps relativement courts. Un tel système se veut une aide à la création pour un éventuel scénariste et ne prétend aucunement remplacer l'humain dans sa tâche première de création.
\end{abstract}

\begin{abstract}
Beyond the traditional methods of screenplay writing, one can well conceive a use of modern computers and particular technics of A.I. as a support for creativity and imagination. The author describes how he has been able to construct a sufficiently strict and rigourous formalisation of narrative theories. This formalisation allows the conception and implantation of an automatic story generation system. This experimental system rests mainly on the particular capacity of computers to generate and to evaluate a large number of alternative stories in a fairly short time. Such a system could be useful as a creative tool for the screenwriter yet cannot pretend in any way to replace the human in his primary task of creation.
\end{abstract}




\section{Un nouvel outil}

Peu de technologies nouvelles ont d'impact signifiant sur le travail d'écriture cinématographique. On comprend que l'apparition successive de nouvelles techniques (le son, la couleur, les lentilles zoom et cinémascope, la 3D simulée, les caméras portatives et synchrones, etc.) a amené une évolution des langages, de la forme du cinéma et même l'apparition de nouveaux «genres» (comme, par exemple, le direct). Plus récemment, l'holographie, la vidéo, la 3D par verres polarisés ou par multiprojections, les multi-films, etc. ont encore accentué le phénomène et influencé la façon dont on «visualise» une histoire pour le cinéma.

Cependant, aucune de ces techniques ne remet en cause fondamentalement le travail du scénariste. L'avènement des machines d'intelligence artificielle risque, au contraire, d'affecter profondément les méthodes de scénarisation.

$\mathrm{Au}$ cours des dernières décennies, plusieurs constatations théoriques portent à croire à la faisabilité d'une machine à générer automatiquement des récits. Des grammaires génératives de Greimas, en passant par les fonctions de Propp, les processus de Bremont, jusqu'au protocole de lecture de Gervais, les chercheurs et formalistes ont souvent développé, à leur insu, des concepts dont on peut dériver des outils pour la génération.

Une telle machine, dotée des outils pertinents, nous permettrait, entre autres, de se définir soi-même comme personnage dans un récit, de choisir la situation historique, géographique, la situation dramatique initiale, etc., et de laisser alors le système automatique générer le scénario pour nous sans que des interventions ultérieures soient requises. Il va de soi qu'une version interactive d'un tel système serait aussi possible, cette dernière permettant à l'usager d'influencer le cours du récit à sa guise un peu à la manière des premiers systèmes hybrides déjà en existence (fictions interactives, livres-réseaux, jeux d'aventures informatisés ou non, etc.). De la même façon, on peut entrevoir, par extrapolation, la génération automatique de films.

Un nombre grandissant de recherches en intelligence artificielle vise à atteindre des objectifs similaires à ceux exposés plus haut ou des étapes intermédiaires indispensables. Le système que je décris dans ce texte s'inscrit dans cette démarche. L'ensemble des développements recherchés dans le domaine vise en général à atteindre un seul objectif commun: en arriver à obtenir un environnement riche et puissant non seulement d'aide à la création mais éventuellement de médiatisation interactive du monde de la création artistique. On vise en particulier, dans bien des cas, la 
mise au point de systèmes permettant la création automatique d'œuvres dramatiques, que ces dernières prennent la forme de films, de pièces de théâtre, d'œuvres littéraires, d'émissions de télévision ou autres.

En ce qui me concerne, un premier objectif à court terme consistait à élaborer un système pouvant générer une séquence d'événements, donc une histoire, plausible d'un point de vue dramatique (autant pour les personnages que pour le récepteur). Toutes les autres considérations inhérentes aux récits, aux langages et aux média ayant été, pour le besoin de cette expérimentation, ignorées.

\section{Quelques prémisses à la théorie du récit}

Pour pouvoir énoncer la possibilité de générer des récits automatiquement, il faut établir un certain nombre d'éléments théoriques dont on pourra tirer un formalisme précis.

De la même façon, un travail de défrichement important s'impose au niveau des théories non seulement des récits automatiques mais des structures de récits en général. J'énonce plus loin quelques-uns des acquis théoriques sur lesquels je me suis basé. Par contre, je n'ai pu que constater l'état embryonnaire des recherches dans le domaine.

Tout récit (...) est la recherche d'une vérité du désir dans son articulation au manque et à la loi (Aumont, Bergala, Marie et Vernet, p. 188).

Toutes les histoires, fondamentalement, ne forment qu'une. Tous les auteurs rejouent la Scène d'Edipe (résolution de l'affrontement du désir et de la loi, de la passion et de la raison). Dans cette optique, il suffit de creuser un écart initial entre un personnage qui a des objectifs et des désirs et son objet de désir quel qu'il soit et on obtient le germe d'un cheminement, d'un itinéraire, donc d'une histoire.

C'est dans la même veine d'idée que les sémiologues en sont venus à constater avec le temps que quels que soient les personnages rencontrés dans les récits, ils accomplissent tous fondamentalement les mêmes actes, et cela en dépit du fait que leurs constituantes spécifiques (âge, sexe, occupations, apparences, état civil, etc.) puissent varier grandement. On remarque le même phénomène pour les lieux et les situations qui, en apparence, varient grandement mais comportent, en réalité, des similitudes de fonctions frappantes.

De là vint la nécessité d'étudier les récits en faisant appel à des fonctions générales. Les chercheurs se sont alors succédé pré- 
sentant chacun une liste de fonctions et prétendant chacun couvrir l'ensemble des actions possibles des récits.

Il est ainsi devenu clair qu'une histoire quelconque peut être identifiée au niveau structurel par des composantes discrètes en nombre limité qui seront des fonctions de transformation et des rôles actantiels.

Par la suite, j'en suis arrivé à deux constatations théoriques importantes. Premièrement, inventer une histoire est un processus distinct et indépendant de l'acte de narration de cette dernière et, deuxièmement, un scénariste éventuel doit terminer la génération d'une séquence d'événements (une histoire) avant de pouvoir en opérer la narration. Cette dernière constatation a son équivalent chez le sémiologue français A. J. Greimas:

L'unité discursive qu'est le récit est à considérer comme un algorithme, c'est-à-dire, comme une succession d'énoncés dont les fonctions-prédicats simulent linguistiquement un ensemble de comportements ayant un but. En tant que succession, le récit possède une dimension temporelle: les comportements qui s'y trouvent étalés entretiennent entre eux des relations d'antériorité et de postériorité.

Le récit, pour avoir un sens, doit être un tout de signification et se présente, de ce fait, comme une structure sémantique simple. Il en résulte que les développements secondaires de la narration, ne trouvant pas leur place dans la structure simple, constituent une couche structurelle subordonnée: la narration, considérée comme un tout, aura donc pour contrepartie une structure hiérarchique (Greimas, p. 34).

Il est donc nécessaire, dans le cadre du processus de génération, de faire une séparation nette entre la génération de la séquence de fonctions ou d'événements et leur «instanciation». Cette philosophie adoptée et conservée dans l'implantation du système élaboré tire sa source, entre autres, des recherches de V. Propp relatées par C. Bremond:

L'Invariant, c'est la fonction que tel ou tel événement, en venant à se produire, remplit dans le cours du récit; le variable, c'est l'affabulation mise en œuvre dans la production et les circonstances de cet événement. Ce qui compte, c'est donc de savoir ce que fait un personnage, quelle fonction il remplit; quant à la question de savoir par qui la chose est faite (homme, animal, être surnaturel, objet), quels moyens cet agent utilise pour la faire (persuasion, duperie, violence, magie, etc.) et dans quelle intention il la fait (pour nuire, rendre service, s'amuser, etc.), elle tombe, nous dit Propp, dans le domaine de l'étude accessoire (Bremond, p. 14). 
J'ajouterai que ce «variable» constitue donc une enveloppe secondaire de l'histoire à générer a posteriori. De plus, j'ai vite constaté de façon empirique que l'on ne pouvait concevoir une structure dramatique plausible et efficace (tant au niveau du spectateur que du récit en soi) sans doter les personnages de structures émotives distinctes pour contrôler leurs attitudes, motivations et actions puisque les émotions, semble-t-il, constituent la clef de voûte du comportement.

À l'aide de sources littéraires diverses, j'ai pu cerner un nombre fini et limité de fonctions déterminantes pour les émotions dans la modélisation des personnages (modélisation qui requiert une structure d'émotions pour établir des «buts», faire de la planification automatique, déterminer des comportements consistants avec la personnalité apparente et déclarée du personnage, etc.).

Les événements constituant un récit peuvent aussi être vus comme des fonctions. Fonctions de transformations faisant le pas d'un état donné à un autre. Le sens de ces fonctions est donc de faire entrer en corrélation tous les différents éléments d'un récit dans la tâche de résolution éventuelle de conflits (Bremont).

$\mathrm{Au}$ cours des années, d'un théoricien à l'autre, ces fonctions ont grandement évolué. Propp, Souriau et Greimas tentèrent en premier d'allouer des fonctions aux personnages d'un récit en «fonction» des actions entreprises.

Ces dernières fonctions peuvent être exécutées de plusieurs façons. Un personnage donné peut en cumuler plusieurs et corrolairement plusieurs personnages peuvent partager une même fonction. Les fonctions ne sont pas toujours attribuées et un même personnage peut changer plusieurs fois de fonctions au cours d'un récit donné.

Vladimir Propp utilisa une approche sensiblement différente en élaborant des fonctions (31) non plus autour de l'action d'un personnage donné mais par rapport à la signification de l'action dans le déroulement de l'intrigue.

On retient des recherches de Propp trois conclusions fondamentales: le nombre de fonctions est limité (au-delà des frontières d'un récit donné) et cela, globalement. Les fonctions constituent les seuls éléments constants et permanents d'un récit à l'autre (au-delà des rôles et des personnages) et finalement la succession des fonctions est toujours identique dans le cadre des sous-séquences internes d'un récit. C'est-à-dire qu'il existe des préséances et prérequis à certaines fonctions (et d'autres corequises et postrequises) qui font que leur succession soit partiellement imposée. Par exemple, on ne peut transgresser un interdit sans s'en être fait imposer un au préalable. Par contre, la 
transgression ne suit pas nécessairement immédiatement l'interdit et peut même ne pas suivre du tout.

En définitive, bien que l'apparition d'une fonction n'est pas déterminée, l'ordre des fonctions ultérieures à celle-ci l'est en grande partie, puisqu'il existe une dépendance entre la situation initiale et les fonctions suivantes. Un système de création automatique de récits est donc libre initialement d'utiliser ou d'omettre les fonctions qu'il désire (parmi celles sans prérequis), mais développe ultérieurement une dépendance certaine dans le séquencement.

Néanmoins, avec les années, plusieurs lacunes significatives devinrent évidentes à l'analyse en profondeur des théories de Propp. Bremont, constatant la nécessité de grouper les fonctions (par constatation de l'impossibilité de permuter ou de supprimer certaines fonctions dans une séquence donnée) et les contraintes de contextes (prérequis et postrequis contraints) en vint à ré-articuler le schéma de fonctions de Propp autour d'entités un peu plus grandes que la fonction tout en restant beaucoup plus petites que la série.

Bremont introduisit les concepts de fonctions étendues, séquences, processus, etc., dans l'élaboration de ses théories narratives. J'ai retenu deux aspects importants de ses conclusions (en plus des éléments de Propp): les concepts de patient-agent, séparation exclusivement dichotomique des rôles possibles réalisables par un actant («acteur»), mais surtout une liste améliorée et étendue des processus (ou fonctions) matérialisables dans des événements donnés.

Le modèle à 29 événements du système implanté (appelé «RÉCIT») constitue une compilation améliorée et adaptée à l'intelligence artificielle des listes de fonctions de Propp (plus de 30) et des processus de Bremont (47). Le tout profondément remanié pour obtenir la rigueur de formalisme nécessaire à une implantation informatique.

\section{D'un système à l'autre}

Nous devons les premiers pas significatifs vers la génération automatique de récits à des recherches sur les représentations de connaissances faites par l'équipe de Schank à Yale (années soixante, soixante-dix et quatre-vingt). Plusieurs logiciels fonctionnels furent développés, entre autres, dans le but de valider ou d'invalider le concept de dépendances conceptuelles alors en développement.

En fait, le but de la plupart de ces expérimentations n'était pas de générer des histoires mais de trouver quel type de connaissances et quelles représentations sont nécessaires pour obtenir 
une telle génération et pour maintenir et modéliser des relations de causalités, des buts, des relations, des motivations, etc.

Le système «RÉCIT» (qui n'utilise pas la représentation de connaissances par dépendances conceptuelles) repose exclusivement sur un algorithme qui explore le champ ou l'ensemble des alternatives possibles. Alors qu'un être humain ne peut que traverser ce champ de façon inefficace et passer possiblement à côté des créations les plus intéressantes, l'algorithme de recherche explore systématiquement toutes les alternatives et peut présenter à l'usager toutes les combinaisons intéressantes.

Bien que dans sa forme actuelle, ce système reste une expérimentation aux capacités timitées, on peut hypothétiquement concevoir une extension plus fonctionnelle de ce dernier qu'un auteur pourrait configurer pour ses besoins spécifiques. Le système pourrait alors explorer le «champ des possibles» dont l'auteur désire faire l'investigation. Il s'agit évidemment dans ce cas-ci de «récits» possibles.

D'autre part, puisque, comme on l'a vu, le récit de fiction, à l'instar des autres formes de récits classiques (contes populaires, légendes, mythes, fables, etc.), repose «sur des structures dont le nombre d'éléments est fini et dont le nombre de combinaisons est limité» (Aumont, Bergala, Marie et Vernet, p. 90), on peut vraisemblablement concevoir qu'un outil de recherche de récits, aussi brut soit-il, puisse réussir, par essais et erreurs, à construire (ou découvrir) des récits décents.

On admet, cependant, qu'une approche systématique est souvent inefficace sinon totalement prohibitive. D'où la nécessité d'ajouter une certaine «intelligence» au processus de recherche. Cette «intelligence» s'incarne sous forme de règles de construction qui vont endiguer le processus de recherche vers une orientation plus efficace.

Plusieurs règles ont ainsi été élaborées en vue de rendre le système fonctionnel. La règle de construction principale, pour le problème qui nous concerne, est celle de plausibilité. Le système doit arriver à un agencement plausible de circonstances. En d'autres termes, la séquence d'événements générée doit être crédible du point de vue de l'usager et plausible du point de vue des motivations et attitudes des personnages.

Autre règle de construction importante: le système demande de définir un état initial des personnages. On qualifie un personnage par l'intermédiaire de primitives choisies parmi trois ensembles: primitives d'émotions (ex.: Paul désire Julie), primitives d'attitudes (ex.: Paul altruiste) et primitives motivationnelles (ex.: Paul est déçu par Julie). Ceci permet à l'usager de définir une orientation globale à l'histoire générée. Cette der- 
nière règle peut donc, en grande partie, se mettre au service d'une dialectique.

Je crois qu'il aurait été possible de concevoir et d'implanter un système de génération de récits fonctionnant à partir d'un «message» à véhiculer (le système «RÉCIT» pourrait être modifié à cette fin par l'addition, entre autres, d'un superviseur intelligent gérant comme un deus ex machina le choix définitif des événements). Cependant, la détermination préliminaire d'un «message» à communiquer ne fait pas partie du processus créatif ordinaire de la plupart des auteurs. Il devient alors artificiel et inadéquat de demander à l'usager de se plier à cette tâche. Il y a de multiples problèmes inhérents à la forme à donner à ce message pour que ce dernier devienne «computérisable», aux problèmes de choix de niveaux de significations symboliques, de métaphorisation, de métonymisation, de choix culturels, de choix de forme, etc.

À l'intérieur du système, chaque événement a, grosso-modo, la forme d'un «script» (tel que défini par R. Schank dans la littérature classique en intelligence artificielle), c'est-à-dire une partie «déclencheur», des « acteurs éventuels», des «actions», etc. Le déclenchement d'un événement (comme le déclenchement d'un script) peut donc être le résultat d'une longue suite de causes et ce dernier événement peut, par la suite, engendrer une suite d'effets. La liste de ces événements est parcourue de façon répétitive par l'algorithme, ce dernier déterminant par une fonction heuristique relativement complexe quel événement est le plus pertinent à déclencher en fonction d'un état donné du monde diégétique artificiel.

Le dernier aspect essentiel du modèle réside justement dans la base de faits communs servant à qualifier les états des personnages. États que l'on peut subdiviser en trois sections correspondant aux trois ensembles de primitives: états émotifs, états des attitudes et états motivationnels.

Ces ensembles sont le résultat d'une compilation (critique et réductive) des listes d'émotions, d'attitudes et de facteurs motivationnels isolés par différents auteurs au cours des années (voir, entre autres, Osgood, Frijda, Bremond). Osgood, parmi ceuxci, affirme le caractère significatif des attitudes dans la prédiction (et donc la détermination) des comportements (p. 198). Ce même auteur a élaboré une liste de 76 tuples (couples constitués d'une primitive et de son contraire) permettant de définir le caractère d'une personne. Les trois ensembles constitués pour le système «RÉCIT» constituent une réduction (et une recompilation) des différentes listes consultées. 
En définitive, le système a essentiellement la structure d'un système expert: un moteur d'inférence, une base de connaissances (contenant l'ensemble des événements possibles, les règles de transformations des émotions, les règles d'évaluation des objectifs, etc.) et une base de faits (contenant les personnages, leurs descriptions et leurs états courants).

Les événements possibles sont en petit nombre. Ils ont été déterminés par compilation de tables établies, entre autres, par des formalistes russes et français (par exemple: méfait, punition, interdiction, transgression, bienfait, etc.). Ces événements ne peuvent être modifiés par l'usager. Ce sont des actes ou des événements non instanciés et le système générateur n'en fait pas l'instanciation. Par exemple, si un personnage $\mathrm{X}$ commet un méfait envers le personnage $Y$, le système générateur, dans un premier temps, ne spécifie pas la nature de ce méfait.

Le processus global est le suivant: tous les personnages changent d'états lorsqu'un événement est déclenché, et tout particulièrement les deux personnages impliqués. Après un événement donné, ce sont les états émotifs des personnages qui déterminent quel événement suivant sera déclenché, quel personnage agira et quel personnage subira l'action.

Une fois toutes les transformations effectuées, le processus d'évaluation-modification peut alors recommencer et cela indéfiniment. Ainsi, les histoires n'ont pas de conclusion en soi et l'usager peut toujours, s'il le désire, demander l'exécution d'un événement suivant ou, s'il le préfère, terminer l'histoire. Ce système-expert «scénariste» est conçu exclusivement comme une aide à la création et, en tant que telle, tend à ne pas imposer de choix créatif.

Il existe un module secondaire au système, un instanciateur, qui permet d'amener l'histoire à un niveau un peu plus avancé de complétion. Ce dernier système est relativement simple à comparer au générateur en soi. Il représente une solution minimale et imparfaite au problème de l'instanciation, mais suffisante pour démontrer expérimentalement que le problème est solvable.

Pour chaque événement possible généré par «RÉCIT», l'instanciateur fait appel à une procédure spécifique d'actions. Pour un événement donné, la procédure pourra aller chercher une nouvelle action ou information dans sa base générale de données, voir à formuler sous forme de texte compréhensible cette action en fonction de la nature de l'événement et du contexte présent et afficher le texte en sortie.

La complexité réelle d'un tel système ne me permet pas d'en exposer toutes les constituantes dans le cadre de ce court article ${ }^{1}$. 


\section{Développements futurs}

L. M. Tard, dans son article sur le livre-minute, énonce: «Un logiciel de conception totale de romans est impensable. Les machines à traiter le texte peuvent tout au plus établir des hypothèses de scénarios, des alternatives de déroulements mais ne remplacent pas l'imagination» (p. 4).

Bien que nous reconnaissons, dans l'état actuel de nos connaissances en intelligence artificielle, que la conception complète d'une œuvre comme un roman (de l'idée au texte fini) soit effectivement impensable, nous n'excluons pas que cet objectif puisse être atteint éventuellement.

En fait, l'objectif susmentionné (établissement d'hypothèses de scénarios et d'alternatives de déroulements) requiert pour être atteint un système d'intelligence artificielle du genre de «RÉCIT», c'est-à-dire un système à base de connaissances d'un tout autre ordre que les machines à traiter le texte.

Ce dernier système, malgré ses limitations certaines, renferme plusieurs qualités non négligeables. Par exemple, l'essentiel des connaissances de «RÉCIT» sont sous formes déclaratives. Ces dernières se prêtent très bien à toutes formes de modifications (manuelles ou automatiques). Un usager peut s'adonner manuellement à de fins réglages de structures d'événements (par l'intermédiaire d'un éditeur) ou un mécanisme automatique d'acquisition de nouvelles connaissances (ou de modifications des anciennes) peut être conçu aux mêmes fins. Il s'agirait alors d'une forme d'apprentissage automatique reposant sur des interactions avec l'usager, ce dernier se prêtant à une évaluation critique des histoires générées.

Autre développement majeur envisageable: le développement d'un mécanisme de narration. Les différents procédés de narration - choix des points de vue, monologues intérieurs, dialogues, suppression d'événements ou altérations de la séquence, retours en arrière, ellipses, enchaînements, modifications du champ narratif, etc. - impliquent l'existence et l'implantation de capacités qui ne sont pas implicitement rattachées au mécanisme fondamental de la génération de récits. Bien que j'aie jugé pour cette dernière raison que je pouvais faire abstraction du problème dans le cadre de mon expérimentation, il faudra éventuellement s'y attaquer car, quoi qu'on en dise, un récit n'est un vrai récit que narré. Il y a encore d'autres niveaux de difficultés qui se rattachent et se superposent à celui de la narration comme, par exemple, les différentes considérations de contextes culturels, de «pathos» dramatiques, de structures sémantiques liées à l'iconicité, au symbolique, etc. 
L'interactivité constitue aussi l'un des développements futurs envisageables pour le système. Cependant, il ne s'agirait pas d'une interactivité de même nature que celle qui caractérise aujourd'hui les jeux d'aventures où c'est l'usager qui finalement construit l'histoire, mais d'une interactivité dialectique entre un créateur éventuel et son outil d'aide à la création. Il s'agit ici d'une malléabilité qui pourrait être donnée à l'outil pour permettre, par exemple, à l'usager de reculer dans une histoire, forcer l'imposition d'un événement ou modifier un état donné pour explorer une hypothèse de déroulement de récit.

Il existe déjà une littérature appréciable concernant les différentes formes de «fiction interactive» informatisée (jeux d'aventures, etc.) ou non informatisée (livres-réseaux où on choisit un chemin variable dans les chapitres ou scènes).

En définitive, il faut bien admettre qu'aucun des systèmes mentionnés ne fait montre de créativité, d'imagination ou même de capacité réelle de génération de récits. Cependant, j'affirme le potentiel prometteur de ce genre de projets et le rôle signifiant qu'ils peuvent jouer, à long terme, sur le travail souvent difficile et ingrat du scénariste.

\section{Université de Montréal}

\section{NOTE}

1 Je préfère référer le lecteur à mon mémoire de maîtrise pour le fonctionnment en détail du système.

\section{OUVRAGES CITÉS}

Aumont, J., Bergala, A., Marie, M., Vernet, M. Esthétique du film. Paris: Nathan, 1983.

Bremond, Claude. Logique du récit. Paris: Seuil, 1973.

Frijda, M. The Emotions. Press Syndicate of the University of Cambridge, 1986.

Gervais, Bertrand, Récits et actions. Montréal: Préambule, 1990.

Greimas, A. J. «Éléments pour une théorie de l'interprétation du récit mythique», «L'Analyse structurale du récit». Numéro spécial de la revue Communication 8 (Paris: Seuil, 1966) p. 34-66.

Greimas, A. J. Du sens et Du sens II. Paris: Seuil, 1983.

Nault, Georges. Modélisation de réactions émotives et application à la génération de récits. Mémoire de maîtrise. Université de Montréal, Département d'informatique et recherches opérationnelles, 1991.

Osgood, Suci, Tannenbaum. The Measurement of Meanings. Urbana, Chicago and London: University of Illinois Press, 1967. 
Propp, Vladimir. Morphologie du conte. Paris: Seuil, 1965 et 1970.

Schank, R., Abelson, R. Scripts Plans Goals and Understanding. Lawrence Erlbaum Associates, Inc., 1977.

Schank, R., Riesbeck, C. Inside Computer Understanding. Lawrence Erlbaum Associates, Inc., 1981.

Tard, L. M. «Le Livre-minute». Les Diplômés (Université de Montréal) no 362 (été 1988) p. 3-5. 